\title{
Multidimensional Evaluation of Traditional Chinese Exercises for Cardiovascular Disease: A Protocol for Systemic Review.
}

\section{NingYang Xu}

Liaoning University of Traditional Chinese Medicine https://orcid.org/0000-0003-3016-5351

\section{Fan Zhang}

Liaoning University of Traditional Chinese Medicine Affiliated Hospital

Hang Li

Liaoning University of Traditional Chinese Medicine

Yue Liu

Liaoning University of Traditional Chinese Medicine Affiliated Hospital

Mei Wang

Liaoning University of Traditional Chinese Medicine Affiliated Hospital

BoYi Dang

Liaoning University of Traditional Chinese Medicine

XueFeng Guan ( $\nabla$ Inzyyxfg@163.com )

https://orcid.org/0000-0003-4869-8623

\section{Research}

Keywords: Traditional Chinese exercises, cardiovascular disease, International Classification of Functioning, Disability and Health (ICF), damage level, Protocol

Posted Date: March 12th, 2020

DOI: https://doi.org/10.21203/rs.3.rs-17025/v1

License: (c) (1) This work is licensed under a Creative Commons Attribution 4.0 International License. Read Full License 


\section{Abstract}

Background: Cardiovascular disease (CVD) remains the No. 1 leading cause of death worldwide and its mortality is still on the rise. Cumulative studies suggest that Traditional Chinese Exercises (TCEs), such as Tai Chi, Baduanjin, and Qi Gong, benefit cardiopulmonary function. In this study, we proposed a metaanalysis protocol to systemically review the benefits of TCEs for CVD using the International Classification of Functioning, Disability and Health (ICF) as the outcome measures.

Methods: Randomized controlled studies on TCEs as a rehabilitation strategy for CVD patients and published till Dec. 2019 will be searched from eleven databases. Benefits of TCEs will be further grouped according to the ICF checklist, the subgroups of TCEs are to address heterogeneity. Study selection and data extraction will be performed independently by two reviewers. Data analysis will be performed using Review Manager software, and the risk of bias will be assessed using Cochrane criteria. Results: Results will be published in a peer-reviewed journal and disseminated electronically and in print.

Conclusion: Using ICF as the reference, this study will provide novel insights on the value of TCEs as a rehabilitation approach for CVD patients. The results will help clinicians to design rehabilitation programs and select appropriate TCEs optimal for CVD patients. The protocol is applying for registration on PROSPERO. Figure A will prove.

\section{Background}

As the leading cause of death in the world, cardiovascular disease (CVD) is as-sociated with not only high morbidity, but increased disability and mortality, posing a major threat to human health, especially of people over 50 years old (1). The World Health Organization (WHO) estimates that approximately 17.9 million people die of CVD each year, accounting for $31 \%$ of the annual global death toll (www.who.int). Corresponding to its growing burden on the global healthcare system, economic evaluation shows that the cost for managing CVD will continue to increase exponentially and the European Heart Networks estimates the current annual cost is approximately 210 billion euros (2-4). At present, medications and surgical interventions are most commonly used for the treatment of late-stage heart diseases. These treatment strategies are often associated with various contraindications, high cost, traumas, and high risk (5) and thus are poorly received by patients. In contrast, exercise-based cardiac rehabilitation (CR) is cost-effective, easy to carry out, and well tolerated by patients. Both the American College of Cardiology Foundation and the European Heart Association acknowledge the importance of cardiac rehabilitation exercise for patients with heart disease $(6,7)$. Studies have shown that active and effective cardiac rehabilitation can save medical costs, high cost-effectiveness, shorter hospital stays, and reduced per capita drug treatment and hospitalization costs $(8,9)$.

The ultimate goal of cardiac rehabilitation is to make the quality of life better for CVD patients. Cumulative studies have clearly demonstrated the benefits of CR in alleviating cardiovascular and/or respiratory symptoms of patients with myocardial infarction, heart failure, or elderly coronary heart 
disease (10) through multiple mechanisms, such as reversing atrial remodeling, improving diastolic function $(11,12)$, increasing exercise endurance and improving patients' life quality $(13,14)$. Exercise training is the most classic and main treatment method for cardiac re-habilitation. It was introduced into clinic more than 50 years ago and its content, regime, and functions have been widely recognized. Exercise training is further di-vided into modern exercise therapies and traditional Chinese exercises (TCEs). Modern exercise therapies mainly focus on aerobic exercise, which, through improving muscular structure and functions, entails both peripheral benefits (such as slowing down heart rate and reducing myocardial oxygen consumption) and central benefits (such as increasing stroke volume, coronary blood flow, and myocardial perfusion), effectively alleviates CVD associated clinical symptoms, and improves patients' capability to live and work (15). TCEs, such as tai chi, qigong, and baduanjin, have been in existence for more than 2,000 years and substantially benefited human health $(16,17)$. TCEs can not only achieve the benefits of modern exercise therapy, but through synchronization of bodily movement and rhythmic breathing, they activate blood flow, strengthening muscles and bones, and consolidating minds (18-21). Therefore, TCE can help improve physiological indicators, physical and chemical results, quality of life, physical function and structural indicators of depression, that is a low-risk, promising intervention $(22,23)$.

Although the mortality of patients with acute heart failure has decreased significantly, most patients surviving the acute attack are left with varying degrees of dysfunctions in the cardiovascular system, respiratory system, exercise endurance, metabolism, and/or mentality. Mounting evidence from the basic and clinical studies on TCEs, such as Tai Chi, Baduanjin, and Wuqinxi showed that these exercises effectively improve the left ventricular ejection fraction (LVEF) (24), abnormal electrocardiogram ST-T changes (25), cardiac index(26), peak heart rate, peak metabolic equivalent, peak oxygen pulse (10), sixmin walking distance (15), Health Survey Short Form (26), cardiopulmonary reserve capacity (27), etc. which have significantly benefited combating anxiety, improving sleep, and lowering blood lipids and pressure $(28,29)$.

In 2001, WHO published ICF, a standard classification skeleton for describing and assessing the interaction between health, health conditions and the environment (30-32). WHO also developed an ICF checklist, which includes 125 categories of information on health and health-related states. Using the ICF checklist, Ewert et al. identified the most common categories of chronic diseases in terms of "body structures", "body functions", "activity and participation" and "environmental factors"; that is, the chronic conditions were assessed based on physiology and anatomy, the individual's performance in life, possible problems and external environmental impacts $(33,34)$.

By far, only one systematic review has been published on TCEs for CVD (35). In that review, the authors focused on physiological and biochemical outcomes. In contrast, here we proposed a protocol that focuses on indicators based on the more comprehensive ICF checklist and explores the impact of TCEs on the rehabilitation status and symptoms of patients with late-stage heart disease during recovery. In addition, ICF checklist-based systemic review is only available for pulmonary heart disease (36), chronic ischemic heart disease (37), chronic ischemic cardiomyopathy (38), and gastric cancer (39); therefore, it 
is time to perform a comprehensive assessment on patients with more common CVD situations, such as coronary heart disease and heart failure. Accordingly, here we performed a meta-analysis on previous studies examining the impacts of TCEs on the body structure, physical function, activity, participation, and environment of patients with heart disease (specifically, coronary heart disease and heart failure) and presented a systemic review to provide a guidance and reference for future treatment of patients with heart disease.

\section{Methods}

\subsection{Study registration}

This study has been registered with the international Prospective Register of Systematic Reviews (PROSPERO), at present, the registration number is still in the application, and the screenshot is the certificate (Appendix Figure A for details).

\subsection{Eligibility criteria 2.2.1. Types of studies}

This study is only including randomized controlled trials (RCTs). No limits on publication dates or any languages restrictions will be placed. All studies are including must also conform the inclusion criteria of the PICOS (population, intervention, comparison, outcome and study) principle.

\subsubsection{Inclusion and exclusion criteria}

Inclusion: First, studies on patients with heart diseases (with clearly stated diagnosis) will be included. Second, we considered only articles that compared an intervention group, that is, a group performing TCEs (eg, tai chi, baduanjin, qigong) with a control group that performed other exercises (eg, strength exercises), that received usual care, or that did not undergo any intervention.

Exclusion: Cohort, observational, case-control, case reports, quasi-RCTs, qualitative and laboratory studies, uncontrolled trials, animal experiments, crossover studies.

\subsubsection{Interventions and comparators}

Studies that compare TCEs as an intervention group with another group receiving or not receiving other interventions. The TCE regimen could be land-or water-based, should aim to prevent or relieve the symptoms of heart disease, regardless of format, duration, frequency or intensity. The control groups could consist of any other forms of exercise, or no intervention.

\subsubsection{Outcome measures \\ 2.2.4.1. Primary outcomes}


According to the latest version of the ICF classification entry, the outcomes of heart disease were evaluated from four aspects, and relevant indicators were extract-ed from the literature.

Physical function

Consciousness function; integral psychosocial function; sleep function; attention function; psychomotor function; emotional function; pain; heart function; vascular function; blood pressure function; blood system function; immune system function; respiratory function; exercise tolerance function; entry function; water, mineral and electrolyte balance function; temperature regulation function; urination function; muscle strength function

Body structure

Structure of the cardiovascular system; structure of the respiratory system

Activities and participation

Master skills; engage in single tasks; engage in multiple tasks; perform daily tasks; control stress and other psychological needs; talk; change the basic posture of the body; maintain a physical posture; move itself; lift and carry objects; move with lower limbs Objects; the use of delicate hands; the use of hands and arms; walking; walking around in different places; using equipment to move around; washing yourself; wearing; eating; drinking; taking care of personal health; doing housework; interpersonal relations; intimacy Relationships; economic transactions; entertainment and leisure.

Environmental factor

Medicines; family and friends; supplies and technologies for personal daily life; supplies and technologies for cultural, recreational and sports; personal care providers and personal assistants; health professionals; health services, institutions and policies.

\subsection{Search methods for studies}

\subsubsection{Electronic searches}

We retrieved articles from the following eleven electronic bibliographic databases from their respective dates of inception to December 2019: PubMed, EMBASE, MEDLINE, The Cochrane Library, Korean Studies Information, Korea Med, Korean National Assembly Library, Chinese National Knowledge Infrastructure (CNKI), Chinese Science and Technology Periodical Database (VIP), Chinese Biomedical Literature Database (CBM), Wan fang Database.

\subsubsection{Other sources}

In order to identify the 'grey' literature/unpublished studies, we will also identify additional relevant studies through review of the WHO International Clinical Trials Registry Platform (ICTRP) (http://apps.who.int/trialsearch/), the Chinese Clinical Trial Registry (http://www.chictr.org.cn/), 
ClinicalTrials.gov (http://clinicaltrials.gov/), and etc. We systematically considered the validity of the identified evidence provided by each piece of 'grey' literature by evaluating the following aspects: the study design, the characteristics of the intervention, and the quality of the methodology, as well as possible key confounding factors; whether it has been correctly registered in the clinical trial registry Registered and documented study number; whether a complete data set of gray literature will be provided; whether the re-search of gray literature has received any ethical approval, etc.

\subsubsection{Search strategy}

Our keywords included 'Baduanjin', 'Tai Chi', 'Wuqinxi', 'Qigong', 'Coronary heart disease', 'myocardial infarction', and 'heart failure'. We used related medical subject terms and synonyms to combine them. (Appendix Table A details the search strategy.)

\subsection{Data collection and analysis 2.4.1. Study inclusion}

Two reviewers (NYX and FZ) independently screened the titles and abstracts of the retrieved studies based on study characteristics, data selection, interventions, methods, results, etc. And reviewed the full text. The differences were resolved through discussions with third and fourth researchers to agree on the ultimate choice of research. The study selection procedures will be presented with reference to the PRISMANMA. (Detailed are listed in Appendix Figure B.)

\subsubsection{Data extraction}

Extracted data will including research project, trial type, year of publication, country/region, inclusion/exclusion criteria, type of heart disease, patient's age, gender, intervention (form of intervention, type, duration of treatment, method), control group, follow-up time, outcome, and adverse events. Coauthors (NYX and FZ) extract data by using standard Excel spreadsheets. Any differences in the data will be reviewed by other team members.

\subsection{Risk of bias assessment of included studies}

According to the Cochrane Handbook (40), the methodological quality of trials will be independently evaluated by two reviewers using the Cochrane risk of bias assessment tool. Any discrepancies will be resolved after rechecking the source papers and further discussion with the third and fourth reviewer. The following criteria will be used:

(1) Sequence generation

(2) Allocation concealment,

(3) Blinding of participants and personnel,

(4) Blinding of outcome assessment 
(5) Incomplete outcome data,

(6) Selective reporting

(7) Other sources of bias.

\subsection{Strategy for data synthesis and statistical analysis 2.6.1. Dealing with missing data}

For studies in which there will miss data or the source of the evaluation scales is unknown, authors will contact through telephone or email. If detailed data still not receive after contacting the author, the available data will be analyzed.

\subsubsection{Data statistical analysis}

For data analysis, we will use Review Manager Software. For continuous data collected using the same measurement scale and continuous data that gave the same result on different measurement scales, the former calculates the weighted mean difference and the $95 \%$ confidence interval $(\mathrm{Cl})$, and the latter has a standardized mean difference of $95 \% \mathrm{Cl}$. For dichotomous outcomes, we will use the hazard ratio with $95 \% \mathrm{Cl}$ and $\mathrm{p}$-value to evaluate the efficacy of traditional exercises on patients with heart disease. For multiple groups of studies, we will analyze each group as an independent group.

\subsubsection{Assessment of heterogeneity}

Random or fixed effect studies based on data analysis results. Heterogeneity of each trial will be assessed by 12 statistics. 12 alone cannot explain the 12 statistic, that is, the proportion of variability in the study. 12 values $>50 \%$ or higher are considered heterogeneous and cannot be ignored. We will explore the source of heterogeneity through subgroup analysis and use forest results to show detection result (40).

\subsubsection{Assessment of reporting biases}

When the number of studies in the systematic review is $\geq 10$, the effect of publication bias on the included studies will be assessed using Egger's test and funnel plots $(40,41)$. If there is a release bias or true heterogeneity occurs, the asymmetry of the funnel graph may be caused.

\subsubsection{Subgroup analysis and investigation of heterogeneity}

Heterogeneity is an important issue to be addressed in this study. So if a sufficient number of studies exist, a subgroup analysis will be performed to explain the heterogeneity in the studies. Subgroup analysis is as follows:

(1) Intervention: TCE classification based on specific TCE method, such as Tai Chi, Ba duanjin, Wu Qin Xi, Yi Jin Jing, and Qigong;

(2) Kinds of heart disease (detailed classification of coronary heart disease; heart failure); 
(3) Experimental design (different exercise types and routine care; different exercise types and other exercise categories);

(4) Exercise intensity: Assess exercise tolerance;

(5) Exercise time: Time of each exercise;

(6) Exercise frequency: Exercise several times a week;

(7) Follow-up (including time and exercise method);

(8) Country/region (East Asia and Western countries) where the study was conducted.

\subsubsection{Sensitivity analysis}

Where appropriate, the stability of our results will be evaluated by assessing methodological quality and changing the statistical model (random effect or fixed effect model) to investigate the stability of the selection process for sensitivity analysis.

\subsubsection{Grading the quality of evidence}

To evaluate the level of confidence on outcomes, we will use the Grading of Recommendations Assessment, Development and Evaluation (GRADE) method (42). Co-authors will conduct the assessment and any discrepancies will be resolved by the third and fourth researchers if essential.

\section{Results}

According to the previous research design, in the work process, the team has begun document retrieval work. Through the search conditions, among the tens of thousands of documents, there are already 23 eligible articles on PubMed. It can be seen that our work is feasible. It will have a significant impact on traditional Chinese exercises and the rehabilitation of patients with heart disease.

\section{Discussion}

This is the protocol for a systematic review to assess the rehabilitation effect and curative effect of TCEs in treating heart disease. Following the protocol, the system will conduct widespread and unbiased analysis and review of 11 databases without language restrictions, and thus allow readers to access studies initially published in East Asian languages that they will if not be unable to read. In contrast to the available systemic review and meta-analysis of TCEs for heart diseases, this study focuses on impacts of TCEs on the rehabilitation of people with heart disease. After analyzing the various groups of exercise intensity, exercise time, exercise frequency, follow-up time and other subgroups, the potential of TCEs in patients with coronary heart disease or heart failure will be assessed comprehensively from the physical structure, physical function, activity, participation, and environmental factors, which provides valuable information on whether TCEs can improve the quality of life and promote the returning of patients to 
society, linking evidence directly to decision-making, and serving as an important reference for the future rehabilitation and treatment of heart disease.

\section{Conclusion:}

It is of great clinical significance if CVD patients could benefit from exercises, such as TCEs during the late rehabilitation phase. However, the lack of comprehensive and systemic evaluation on the value of TCEs has impeded their promotions and widespread use among CVD patients. This paper, by proposing a meta-analysis protocol using ICF checklist as the evaluation criteria, will provide evidence-based information on the efficacy and safety of TCEs for CVD.

\section{Abbreviations}

1. 1.International Classification of Functioning, Disability and Health (ICF)

2. cardiovascular disease (CVD)

3. Traditional Chinese Exercises (TCEs)

4. 4.World Health Organization (WHO)

5. Grading of Recommendations Assessment, Development and Evaluation (GRADE)

6. randomized controlled trials (RCTs).

\section{Declarations}

Ethics approval and consent to participate

Not applicable

Consent for publication

Not applicable

Availability of data and materials

Not applicable

Competing interests

The authors declare that they have no competing interests

\section{Funding}

This work was supported by the World traditional therapy research and national cooperation demonstration base by the State Administration of Traditional Chinese Medicine [Grant numbers GJZX2016022]. 


\section{Authors' contributions}

XFG was responsible for the direction of the research project. NYX and FZ made critical contributions to the study concept and design, NYX and HL designed the literature search, YL perfected the design and development of research programs. NYX, FZ and BYD will undertake study selection, data extraction. MW and XFG were responsible for evaluating the eligibility of studies. NYX and FZ undertake analysis, interpretation and report writing. All authors read and approved the final manuscript.

\section{Acknowledgements}

Not applicable

\section{References}

1. Writing Group M, Mozaffarian D, Benjamin EJ, Go AS, Arnett DK, Blaha MJ, et al. Heart Disease and Stroke Statistics-2016 Update: A Report From the American Heart Association. Circulation. 2016;133(4):e38-360.

2. Anand SS, Yusuf S. Stemming the global tsunami of cardiovascular disease. Lancet. 2011;377(9765):529-32.

3. Timmis A, Townsend N, Gale CP, Torbica A, Lettino M, Petersen SE, et al. European Society of Cardiology: Cardiovascular Disease Statistics 2019. Eur Heart J. 2020;41(1):12-85.

4. Tofield A. European cardiovascular disease statistics 2012 summary. Eur Heart J. 2013;34(15):1086.

5. Golwala H, Pandey A, Ju C, Butler J, Yancy C, Bhatt DL, et al. Temporal Trends and Factors Associated With Cardiac Rehabilitation Referral Among Patients Hospitalized With Heart Failure: Findings From Get With The Guidelines-Heart Failure Registry. J Am Coll Cardiol. 2015;66(8):917-26.

6. Jacobs AK, Kushner FG, Ettinger SM, Guyton RA, Anderson JL, Ohman EM, et al. ACCF/AHA clinical practice guideline methodology summit report: a report of the American College of Cardiology Foundation/American Heart Association Task Force on Practice Guidelines. J Am Coll Cardiol. 2013;61(2):213-65.

7. McMurray JJ, Adamopoulos S, Anker SD, Auricchio A, Bohm M, Dickstein K, et al. ESC guidelines for the diagnosis and treatment of acute and chronic heart failure 2012: The Task Force for the Diagnosis and Treatment of Acute and Chronic Heart Failure 2012 of the European Society of Cardiology. Developed in collaboration with the Heart Failure Association (HFA) of the ESC. Eur J Heart Fail. 2012;14(8):803-69.

8. Shields GE, Wells A, Doherty P, Heagerty A, Buck D, Davies LM. Cost-effectiveness of cardiac rehabilitation: a systematic review. Heart. 2018;104(17):1403-10.

9. Lopez-Montecinos P, Rebolledo SJ, Gomez LJ. [Cost effectiveness of a theoretical cardiac rehabilitation program after myocardial infarction]. Rev Med Chil. 2016;144(4):456-64.

10. Nery RM, Zanini M, de Lima JB, Buhler RP, da Silveira AD, Stein R. Tai Chi Chuan improves functional capacity after myocardial infarction: A randomized clinical trial. Am Heart J. 2015;169(6):854-60. 
11. Edelmann F, Gelbrich G, Dungen HD, Frohling S, Wachter R, Stahrenberg R, et al. Exercise training improves exercise capacity and diastolic function in patients with heart failure with preserved ejection fraction: results of the Ex-DHF (Exercise training in Diastolic Heart Failure) pilot study. J Am Coll Cardiol. 2011;58(17):1780-91.

12. Haykowsky M, Scott J, Esch B, Schopflocher D, Myers J, Paterson I, et al. A meta-analysis of the effects of exercise training on left ventricular remodeling following myocardial infarction: start early and go longer for greatest exercise benefits on remodeling. Trials. 2011;12:92.

13. Ghanbari-Firoozabadi M, Rahimianfar AA, Reza Vafaii Nasab M, Namayandeh SM, Emami M, Boostani $F$, et al. A study of the effect of cardiac rehabilitation on heart failure patients' life quality. $J$ Med Life. 2014;7(1):51-4.

14. Lyamina NP, Karpova ES, Kotel'Nikova EV, Bizyaeva EA. Preconditioning at the stages of invasive and rehabilitative treatment of patients with coronary heart disease. Klin Med. 2015;93(3):14-20.

15. Mahendra M, Sk S, Desai N, Bs J, Pa M. Evaluation for airway obstruction in adult patients with stable ischemic heart disease. 2018:266-71.

16. Chan JS, Ho RT, Chung KF, Wang CW, Yao TJ, Ng SM, et al. Qigong exercise alleviates fatigue, anxiety, and depressive symptoms, improves sleep quality, and shortens sleep latency in persons with chronic fatigue syndrome-like illness. Evid Based Complement Alternat Med. 2014;2014:106048.

17. Guo Y, Qiu P, Liu T. Tai Ji Quan: An overview of its history, health benefits, and cultural value. JSHS. 2014;3(1):3-8.

18. Cheng FK. Effects of Baduanjin on mental health: a comprehensive review. J Bodyw Mov Ther. 2015;19(1):138-49.

19. Stevens JA, Voukelatos A, Ehrenreich H. Preventing falls with Tai Ji Quan: A public health perspective. J Sport Health Sci. 2014;3(1):21-6.

20. Wen J, Lin T, Cai Y, Chen Q, Chen Y, Ren Y, et al. Baduanjin Exercise for Type 2 Diabetes Mellitus: A Systematic Review and Meta-Analysis of Randomized Controlled Trials. Evid Based Complement Alternat Med. 2017;2017:8378219.

21. Li F, Harmer PA. Tai Ji Quan: From traditional applications to contemporary practice. J Sport Health Sci. $2014 ; 3(1): 1-2$.

22. Hartley L, Flowers N, Lee MS, Ernst E, Rees K. Tai chi for primary prevention of cardiovascular disease. Cochrane Database Syst Rev. 2014(4):CD010366.

23. Jiang Y, Zou J. Analysis of the TCM theory of traditional Chinese health exercise. J Sport Health Sci. 2013;2(4):204-8.

24. Acar RD, Bulut M, Ergün S, Yesin M, Ak?akoyun M. Evaluation of the effect of cardiac rehabilitation on left atrial and left ventricular function and its relationship with changes in arterial stiffness in patients with acute myocardial infarction. Echocardiography. 2015;32(3):443-7.

25. Bouts AM, Brackman L, Martin E, Subasic AM, Potkanowicz ES. The Accuracy and Validity of iOSBased Heart Rate Apps During Moderate to High Intensity Exercise. Int J Exerc Sci. 2018;11(7):53340. 
26. Salmoirago-Blotcher E, Wayne PM, Dunsiger S, Krol J, Breault C, Bock BC, et al. Tai Chi Is a Promising Exercise Option for Patients With Coronary Heart Disease Declining Cardiac Rehabilitation. J Am Heart Assoc. 2017;6(10).

27. Avila A, Claes J, Goetschalckx K, Buys R, Azzawi M, Vanhees L, et al. Home-Based Rehabilitation With Telemonitoring Guidance for Patients With Coronary Artery Disease (Short-Term Results of the TRiCH Study): Randomized Controlled Trial. J Med Internet Res. 2018;20(6):e225.

28. Xiao C, Yang Y, Zhuang Y. Effect of Health Qigong Ba Duan Jin on Blood Pressure of Individuals with Essential Hypertension. J Am Geriatr Soc. 2016;64(1):211-3.

29. Kim H-K, Takahashi M, Konishi M, Tabata H, Nishimaki M, Xiang M, et al. P-46 Effects of increased daily physical activity on blood lipids and blood pressure in older adults. Brit J Sport Med. 2016;50:A57-A8.

30. Svestkova O. International classification of functioning, disability and health of World Health Organization (ICF). Prague Med Rep. 2008;109(4):268-74.

31. Ustun TB, Chatterji S, Bickenbach J, Kostanjsek N, Schneider M. The International Classification of Functioning, Disability and Health: a new tool for understanding disability and health. Disabil Rehabil. 2003;25(11-12):565-71.

32. Staff. International Classification of Functioning, Disability, and Health: World Health Organization; 2008.

33. Ewert T, FuessI M, Cieza A, Andersen C, Chatterji S, Kostanjsek N, et al. Identification of the most common patient problems in patients with chronic conditions using the ICF checklist. J Rehabil Med. 2004(44 Suppl):22-9.

34. Stucki G. International Classification of Functioning, Disability, and Health (ICF): a promising framework and classification for rehabilitation medicine. Am J Phys Med Rehabil. 2005;84(10):73340.

35. Wang XQ, Pi YL, Chen PJ, Liu Y, Wang R, Li X, et al. Traditional Chinese Exercise for Cardiovascular Diseases: Systematic Review and Meta-Analysis of Randomized Controlled Trials. J Am Heart Assoc. 2016;5(3):e002562.

36. Boldt C, Grill E, Wildner M, Portenier L, Wilke S, Stucki G, et al. ICF Core Set for patients with cardiopulmonary conditions in the acute hospital. Disabil Rehabil. 2005;27(7-8):375-80.

37. You M, Wen F, Xu W, Tiantong Y. Modelling of the ICF core sets for chronic ischemic heart disease using the LASSO model in Chinese patients. Health Qual Life Ou. 2018;16(1):139.

38. Cieza A, Stucki A, Geyh S, Berteanu M, Quittan M, Simon A, et al. ICF Core Sets for chronic ischaemic heart disease. J Rehabil Med. 2004(44 Suppl):94-9.

39. Castaneda L, Bergmann A, Bahia L. The International Classification of Functioning, Disability and Health: a systematic review of observational studies. Rev Bras Epidemiol. 2014;17(2):437-51.

40. Higgins JP, Green S. Cochrane Handbook for Systematic Reviews of Interventions Version 5.1.0 The Cochrane Collaboration2011 [updated March 2011. Available from: http://training.cochrane.org/handbook. 
41. Doncaster CP, Spake R. Correction for bias in meta-analysis of little-replicated studies. Methods Ecol Evol. 2017;9(3):1-11.

42. Balshem H, Helfand M, Schunemann HJ, Oxman AD, Kunz R, Brozek J, et al. GRADE guidelines: 3 . Rating the quality of evidence. J Clin Epidemiol. 2011;64(4):401-6.

\section{Supplementary Files}

This is a list of supplementary files associated with this preprint. Click to download.

- AppendixFigureB.docx

- AppendixFigureA.docx

- PRISMAchecklist.doc

- AppendixTableA.docx 